\title{
ON GEOMETRY OF FINSLER CAUSALITY: FOR CONVEX CONES, THERE IS NO AFFINE-INVARIANT LINEAR ORDER (SIMILAR TO COMPARING VOLUMES)
}

\author{
Olga Kosheleva \\ Ph.D. (Phys.-Math.), Associate Professor, e-mail: olgak@utep.edu \\ Vladik Kreinovich \\ Ph.D. (Phys.-Math.), Professor, e-mail:vladik@utep.edu \\ University of Texas at El Paso, El Paso, Texas 79968, USA
}

\begin{abstract}
Some physicists suggest that to more adequately describe the causal structure of space-time, it is necessary to go beyond the usual pseudoRiemannian causality, to a more general Finsler causality. In this general case, the set of all the events which can be influenced by a given event is, locally, a generic convex cone, and not necessarily a pseudo-Reimannian-style quadratic cone. Since all current observations support pseudo-Riemannian causality, Finsler causality cones should be close to quadratic ones. It is therefore desirable to approximate a general convex cone by a quadratic one. This can be done if we select a hyperplane, and approximate intersections of cones and this hyperplane. In the hyperplane, we need to approximate a convex body by an ellipsoid. This can be done in an affine-invariant way, e.g., by selecting, among all ellipsoids containing the body, the one with the smallest volume; since volume is affine-covariant, this selection is affine-invariant. However, this selection may depend on the choice of the hyperplane. It is therefore desirable to directly approximate the convex cone describing Finsler causality with the quadratic cone, ideally in an affine-invariant way. We prove, however, that on the set of convex cones, there is no affine-covariant characteristic like volume. So, any approximation is necessarily not affine-invariant.
\end{abstract}

Keywords: space-time geometry, Finsler spaces, causality.

\section{Formulation of the Corresponding Physical Problem: Analysis of Finsler Causality Relations}

Geometric description of physical causality: a brief reminder (for details, see, e.g., [5]). In Newton's mechanics, a space-time event $(t, x)$ can causally influence an event $\left(t^{\prime}, x^{\prime}\right)$ if and only if $t \leqslant t^{\prime}$. In Special Relativity Theory, a space-time event $(t, x)$, with $x=\left(x_{1}, \ldots, x_{m}\right)$, can causally influence an event $\left(t^{\prime}, x^{\prime}\right)$ with $x^{\prime}=\left(x_{1}^{\prime}, \ldots, x_{m}^{\prime}\right)$ if the difference vector

$$
\left(\Delta t, \Delta x_{1}, \ldots, \Delta x_{m}\right) \stackrel{\text { def }}{=}\left(t^{\prime}-t, x_{1}^{\prime}-x_{1}, \ldots, x_{m}^{\prime}-x_{m}\right)
$$


belongs to the quadratic cone

$$
C=\left\{\left(\Delta t, \Delta x_{1}, \ldots, \Delta x_{m}\right): \Delta t \geqslant 0 \&(\Delta t)^{2}-\left(\Delta x_{1}\right)^{2}-\ldots-\left(\Delta x_{m}\right)^{2} \geqslant 0\right\} .
$$

According to the General Relativity Theory, similar cones describe local causality: a space-time event $(t, x)$, with $x=\left(x_{1}, \ldots, x_{m}\right)$, can causally influence an event $(t+d t, x+d x)$ with $d x=\left(d x_{1}, \ldots, d x_{m}\right)$ if the difference vector $\left(d t, d x_{1}, \ldots, d x_{m}\right)$ belongs to the cone

$$
C=\left\{\left(d t, d x_{1}, \ldots, d x_{m}\right): d t \geqslant 0 \&(d t)^{2}-\left(d x_{1}\right)^{2}-\ldots-\left(d x_{m}\right)^{2} \geqslant 0\right\} .
$$

Finsler causality. Some physical theories use more general - not necessarily quadratic - convex cones to describe causality; see, e.g., $[1,4,6]$. This generalization of the usual causality is known as Finsler causality.

It is desirable to approximate Finsler causality with the usual one. As of now, all observations are consistent with the usual causality relation, namely with the locally quadratic causality cones of General Relativity theory. Thus, even if the actual causality relation is a Finsler one, it is close to the usual quadratic one. So, to be able to efficiently deal with Finsler causality relations, it is therefore desirable to be able to approximate Finsler causality cones with quadratic ones. This way, we will be able to use the formulas corresponding to the usual quadratic causality as the first approximation, and thus to concentrate our analysis on the empirically small - differences between these causality relations.

How can we find such an approximation?

A possible approach to the desired approximation. A convex cone is uniquely determined by its intersection with a hyperplane - which is a convex body. For the quadratic cone that corresponds to the usual causality relation, this intersection is a bounded quadratic body, i.e., an ellipsoid - and vice versa, for each ellipsoid, the corresponding cone is a quadratic cone. For general convex cones, the intersection is a generic convex body.

So, to approximate a generic convex cone by a quadratic one, it is sufficient to approximate a general convex body by an ellipsoid. This is indeed possible. For example, to every bounded convex body $B \subseteq \mathbb{R}^{n}$ with a non-empty interior, we can associate a unique ellipsoid $E$ enclosing this body: namely, out of all ellipsoids $E$ that contain the set $B$, we can select the ellipsoid $E_{0}$ with the smallest possible volume $V(E)$ (see, e.g., $[2,3]$ ):

$$
V\left(E_{0}\right)=\min \{V(E): E \text { is an ellipsoid and } B \subseteq E\} .
$$

This definition makes perfect sense, since for ellipsoids, $E \subseteq E^{\prime}$ and $E \neq E^{\prime}$ imply that $V(E)<V\left(E^{\prime}\right)$. Thus, the fact that the ellipsoid $E_{0}$ has the minimal possible volume implies that there is no sub-ellipsoid $E^{\prime} \subseteq E_{0}$ that contains the original body $B$.

Strictly speaking, to describe the volume, we need to fix (affine) coordinates in the corresponding hyperplane. However, while the actual values of the volume 
depend on the coordinates, the resulting ellipsoid is the same no matter what coordinates we use. The reason for this is that the volume is affine-covariant: for any affine transformation $T$ that describes the transition to new affine coordinates, and for every ellipsoid $E$, we have $V(T E)=c(T) \cdot V(E)$ for some constant $c(T)$. As a result, for every two ellipsoids $E$ and $E^{\prime}$, we have $V(E)<V\left(E^{\prime}\right) \Leftrightarrow V(T E)<$ $V\left(T E^{\prime}\right)$, i.e., which ellipsoid has larger volume and which has smaller volume does not depend on the choice of the coordinates.

Limitations of the usual approximation, and the resulting problem. The problem with the above approximation idea is that to follow this idea, we must first choose a hyperplane, and there is no guarantee that for a different hyperplane, we will not get a different approximation.

To make the approximation more physically meaningful, it is therefore desirable to avoid such an arbitrary choice, and instead of approximating a body by an ellipsoid, to directly approximate a general convex cone $C$ by a quadratic cone $Q$. Out of all possible quadratic cones $Q$ that contain $C$, it is desirable to select the "smallest" one in the sense of an appropriate total order $\leqslant$ on the class of all quadratic ellipsoids. This selection should not depend on the choice of coordinates. To guarantee that, it is desirable to make sure that the corresponding order is affine-covariant, i.e., that $Q<Q^{\prime}$ if and only if $T Q<T Q^{\prime}$.

What we do in this paper. In this paper, we show that for quadratic cones, there are no affine-invariant volume-like characteristics. Moreover, we show that there is no affine-invariant ordering relation between quadratic cones.

The physical meaning of this result is that it is not possible to approximate Finsler causality by quadratic causality in an affine-invariant way.

\section{Definitions and the Main Result}

Definition 1. Let $n \geqslant 2$.

- By an affine transformation $T: \mathbb{R}^{n} \rightarrow \mathbb{R}^{n}$, we mean a reversible mapping of the type $x_{i} \rightarrow \sum_{j=1}^{n} t_{i j} \cdot x_{j}$.

- By a quadratic cone, we mean an image $T Q_{0}$ of the standard quadratic cone

$$
Q_{0}=\left\{\left(x_{1}, \ldots, x_{n}\right): x_{1} \geqslant 0 \& x_{1}^{2} \geqslant x_{2}^{2}+\ldots+x_{n}^{2}\right\}
$$

under an affine transformation.

Notation. For each $n \geqslant 2$, let $\mathcal{Q}_{n}$ denote the set of all $n$-dimensional quadratic cones.

Definition 2. By a linear (total) pre-order we mean a transitive relation $\leqslant$ for which, for every two objects $a$ and $b$, we have $a \leqslant b$ or $b \leqslant a$.

Comment. For some objects $a$ and $b$, it is possible to have both $a \leqslant b$ and $b \leqslant a$. For example, for the corresponding relation on the ellipsoids $E \leqslant E^{\prime} \Leftrightarrow V(E) \leqslant V\left(E^{\prime}\right)$, 
when we have two ellipsoids $E$ and $E^{\prime}$ of the same volume, then we have both $E \leqslant E^{\prime}$ and $E^{\prime} \leqslant E$.

Notation. We will denote:

- the situation when $a \leqslant b$ and $b \nless a$ by $a<b$, and

- the situation when $a \leqslant b$ and $b \leqslant a$ by $a \sim b$.

Definition 3. Let $n \leqslant 2$.

- A linear pre-order on the set $\mathcal{Q}_{n}$ is called $\subseteq$-consistent if $Q \subseteq Q^{\prime}$ and $Q \neq Q^{\prime}$ imply that $Q<Q^{\prime}$.

- A linear pre-order on the set $\mathcal{Q}_{n}$ is called affine-invariant if for each affine transformation $T, Q \leqslant Q^{\prime}$ implies $T Q<T Q^{\prime}$.

Proposition. For each $n \geqslant 2$, no linear order on the set $\mathcal{Q}_{n}$ of all $n$-dimensional quadratic cones is both $\subseteq$-consistent and affine-invariant.

Comment. The formulation of this proposition of ours first appeared in Geombinatorics journal.

Proof. We will prove this result by contradiction. Let us assume that a linear order $\leqslant$ is a $\subseteq$-consistent and affine-invariant linear order on the set $\mathcal{Q}_{n}$, and let us deduce a contradiction from this assumption.

$1^{\circ}$. Let us first consider the case $n=2$.

$1.1^{\circ}$. Let us first consider the following quadratic cone

$$
Q_{1} \stackrel{\text { def }}{=}\left\{\left(x_{1}, x_{2}\right): x_{1} \geqslant 0 \& x_{2} \geqslant 0\right\} \text {. }
$$

An affine transformation $T_{1}:\left(x_{1}, x_{2}\right) \rightarrow\left(-x_{2}, x_{1}\right)$ maps this cone into

$$
T_{1} Q_{1}=\left\{\left(x_{1}, x_{2}\right): x_{1} \leqslant 0 \& x_{2} \geqslant 0\right\} .
$$

If we apply the same transformation $T_{1}$ again, we get

$$
\begin{aligned}
& T_{1}^{2} Q_{1}=\left\{\left(x_{1}, x_{2}\right): x_{1} \leqslant 0 \& x_{2} \leqslant 0\right\}, \\
& T_{1}^{3} Q_{1}=\left\{\left(x_{1}, x_{2}\right): x_{1} \geqslant 0 \& x_{2} \leqslant 0\right\},
\end{aligned}
$$

and $T_{1}^{4} Q_{1}=Q_{1}$.

$1.2^{\circ}$. Since $\leqslant$ is a linear order, we have either $Q_{1} \leqslant T_{1} Q_{1}$ or $T_{1} Q_{1} \leqslant Q_{1}$. Let us consider these two possibilities one by one.

1.2.1 . If $Q_{1} \leqslant T_{1} Q_{1}$, then, since the order $\leqslant$ is affine-invariant, we get $T_{1} Q_{1} \leqslant T_{1}^{2} Q_{1}, T_{1}^{2} Q_{1} \leqslant T_{1}^{3} Q_{1}$, and $T_{1}^{3} Q_{1} \leqslant Q_{1}$. Thus, by transitivity, we get $T_{1} Q_{1} \leqslant Q_{1}$ and hence,

$$
Q_{1} \sim T_{1} Q_{1} .
$$


1.2.2 $2^{\circ}$ If $T_{1} Q_{1} \leqslant Q_{1}$, then we similarly get $Q_{1} \leqslant T_{1} Q_{1}$ and thus, $Q_{1} \sim T_{1} Q_{1}$. So, in both cases, we have $Q_{1} \sim T_{1} Q_{1}$.

$1.3^{\circ}$. Let us now consider a different affine transformation

$$
T_{2}\left(x_{1}, x_{2}\right)=\left(x_{1}+x_{2}, x_{2}\right) .
$$

For this transformation, $T_{2} Q_{1}=\left\{\left(x_{1}, x_{2}\right): 0 \leqslant x_{2} \leqslant x_{1}\right\}$ and

$$
T_{2} T_{1} Q_{1}=\left\{\left(x_{1}, x_{2}\right): x_{2} \geqslant 0 \& x_{1} \leqslant x_{2}\right\} .
$$

Since $Q_{1} \sim T_{1} Q_{1}$, we have $T_{2} Q_{1} \sim T_{2} T_{1} Q_{1}$.

On the other hand, since $T_{2} Q_{1} \subseteq Q_{1}$ and $T_{2} Q_{1} \neq Q_{1}$, we have $T_{2} Q_{1}<Q_{1}$. Similarly, since $T_{1} Q_{1} \subseteq T_{2} T_{1} Q_{1}$ and $T_{1} Q_{1} \neq T_{2} T_{1} Q_{1}$, we have $T_{1} Q_{1}<T_{2} T_{1} Q_{1}$. From $T_{2} Q_{1}<Q_{1}, Q_{1} \sim T_{1} Q_{1}$, and $T_{1} Q_{1}<T_{2} T_{1} Q_{1}$, we conclude that

$$
T_{2} Q_{1}<T_{2} T_{1} Q_{1}
$$

which contradicts to the previous conclusion that $T_{2} Q_{1} \sim T_{2} T_{1} Q_{1}$. This contradiction shows that for $n=2$, indeed, no $\subseteq$-consistent and affine-invariant is possible on the class $\mathcal{Q}_{n}$.

$2^{\circ}$. Let us now consider the case $n>2$. In this case, we consider a cone

$$
Q_{1}=\left\{\left(x_{1}, \ldots, x_{n}\right): x_{1} \geqslant 0 \& x_{1} \cdot x_{2} \geqslant x_{3}^{2}+\ldots+x_{n}^{2}\right\},
$$

and transformations

$$
T_{1}\left(x_{1}, x_{2}, x_{3}, \ldots, x_{n}\right)=\left(-x_{2}, x_{1}, x_{3}, \ldots, x_{n}\right)
$$

and

$$
T_{2}\left(x_{1}, x_{2}, x_{3}, \ldots, x_{n}\right)=\left(x_{1}+x_{2}, x_{2}, x_{3}, \ldots, x_{n}\right) .
$$

Thus, the inverse transformations take the form

$$
T_{1}^{-1}\left(x_{1}^{\prime}, x_{2}^{\prime}, x_{3}^{\prime}, \ldots, x_{n}^{\prime}\right)=\left(x_{2}^{\prime},-x_{1}^{\prime}, x_{3}^{\prime}, \ldots, x_{n}^{\prime}\right)
$$

and

$$
T_{2}^{-1}\left(x_{1}^{\prime}, x_{2}^{\prime}, x_{3}^{\prime}, \ldots, x_{n}^{\prime}\right)=\left(x_{1}^{\prime}-x_{2}^{\prime}, x_{2}^{\prime}, x_{3}^{\prime}, \ldots, x_{n}^{\prime}\right) .
$$

Here too, $T_{1}^{4} Q_{1}=Q_{1}$, thus $Q_{1} \sim T_{1} Q_{1}$. The set $T_{2} Q_{1}=\left\{x^{\prime}: T_{2}^{-1} x^{\prime} \in Q_{1}\right\}$ has the form

$$
T_{2} Q_{1}=\left\{\left(x_{1}, \ldots, x_{n}\right): x_{1}-x_{2} \geqslant 0 \&\left(x_{1}-x_{2}\right) \cdot x_{2} \geqslant x_{3}^{2}+\ldots+x_{n}^{2}\right\} .
$$

The corresponding inequality

$$
\left(x_{1}-x_{2}\right) \cdot x_{2} \geqslant x_{3}^{2}+\ldots+x_{n}^{2}
$$


is equivalent to

$$
x_{1} \cdot x_{2}-x_{2}^{2} \geqslant x_{3}^{2}+\ldots+x_{n}^{2}
$$

and thus, implies that

$$
x_{1} \cdot x_{1} \geqslant x_{1} \cdot x_{2}-x_{2}^{2} \geqslant x_{3}^{2}+\ldots+x_{n}^{2},
$$

so $T_{2} Q_{1} \subseteq Q_{1}$ and $T_{2} Q_{1} \neq Q_{1}$ and hence, $T_{2} Q_{1}<Q_{1}$.

Similarly, the set $T_{2} T_{1} Q_{1}$ consists of all the points $\left(x_{1}, \ldots, x_{n}\right)$ for which

$$
-x_{2} \cdot\left(x_{1}-x_{2}\right) \geqslant x_{3}^{2}+\ldots+x_{n}^{2},
$$

i.e., for which

$$
-x_{2} \cdot x_{1}+x_{2}^{2} \geqslant x_{3}^{2}+\ldots+x_{n}^{2}
$$

So, if $\left(x_{1}, \ldots, x_{n}\right) \in T_{1} Q_{1}$, i.e., if

$$
-x_{2} \cdot x_{1} \geqslant x_{3}^{2}+\ldots+x_{n}^{2}
$$

then

$$
-x_{2} \cdot x_{1}+x_{2}^{2} \geqslant x_{3}^{2}+\ldots+x_{n}^{2}
$$

and thus, $x \in T_{2} T_{1} Q_{1}$. So here too, $T_{1} Q_{1} \subseteq T_{2} T_{1} Q_{1}$ and $T_{1} Q_{1} \neq T_{2} T_{1} Q_{1}$, hence $T_{1} Q_{1}<T_{2} T_{1} Q_{1}$.

Similarly to Part 1 of this proof, from $T_{2} Q_{1}<Q_{1}, Q_{1} \sim T_{1} Q_{1}$, and

$$
T_{1} Q_{1}<T_{2} T_{1} Q_{1}
$$

we conclude that $T_{2} Q_{1}<T_{2} T_{1} Q_{1}$, which contradicts to the previous conclusion that $T_{2} Q_{1} \sim T_{2} T_{1} Q_{1}$. This contradiction shows that for any $n$, no $\subseteq$-consistent and affine-invariant is possible on the class $\mathcal{Q}_{n}$.

\section{Acknowledgments}

This work was supported in part by the National Science Foundation grants HRD-0734825 and HRD-1242122 (Cyber-ShARE Center of Excellence) and DUE0926721. The authors are thankful to D. G. Pavlov and to all the participants of the 5th International Conference on Finsler Extensions of Relativity Theory FERT, (Moscow-Fryazino, Russia) for valuable discussions.

\section{REFERENCES}

1. Asanov S.S. Finsler Geometry, Relativity and Gauge Theories. Springer Verlag, Berin, Heidelberg, New York, 1985.

2. Busemann H. Metric Methods of Finsler Spaces and in the Foundations of Geometry. Princeton University Press, Princeton, New Jersey, 1943.

3. Busemann H. Geometry of Geodesics. Dover Publ., New York, 2005. 
4. Chang Z. and Li V. Modified Friedmann model in Randers-Finsler space of approximate Berwald type as a possible alternative to dark energy hypothesis. Physics Letters, 2009, vol. B676, pp. 173-176.

5. Misner C.W., Thorne K.S., and Wheeler J.A. Gravitation. Freeman Publ., San Francisco, California, 1973.

6. Pavlov D.G., Atanasiu Gh., and Balan V. (eds.) Space-Time Structure. Algebra and Geometry, Lilia-Print, Moscow, 2007.

\title{
О ГЕОМЕТРИИ ПРИЧИННОСТИ ФИНСЛЕРА: ДЛЯ ВЫПУКЛЫХ КОНУСОВ НЕ СУЩЕСТВУЕТ АФФИННО-ИНВАРИАНТНОГО ЛИНЕЙНОГО ПОРЯДКА (АНАЛОГИЧНОГО СРАВНЕНИЮ ОБЪЁМОВ)
}

\author{
О. Кошелева \\ к.ф.-м.н., доцент, е-mail: olgak@utep.edu \\ В. Крейнович \\ к.ф.-м.н., профессор, е-mail: vladik@utep.edu \\ Техасский университет в Эль Пасо, США
}

\begin{abstract}
Аннотация. Некоторые физики предполагают, что для более адекватного описания причинной структуры пространства-времени необходимо выйти за пределы обычной псевдоримановой причинности и перейти к более общей причинности Финслера. В этом общем случае множество всех событий, на которые может влиять данное событие, является локально выпуклым конусом общего типа, а не обязательно квадратичным конусом псевдориманова типа. Поскольку все текущие наблюдения подтверждают псевдориманову причинность, конусы причинности Финслера должны быть близки к квадратичным. Поэтому желательно аппроксимировать общий выпуклый конус квадратичным. Это можно сделать, если мы выберем гиперплоскость и аппроксимируем пересечения конусов и этой гиперплоскости. В гиперплоскости нам нужно аппроксимировать выпуклое тело эллипсоидом. Это можно сделать аффинно-инвариантным способом, например, выбрав среди всех эллипсоидов, содержащих тело, тот, который имеет наименьший объем; так как объем является аффинно-ковариантным, этот отбор является аффинно-инвариантным. Однако, этот отбор может зависеть от выбора гиперплоскости. Поэтому желательно непосредственно аппроксимировать выпуклый конус, описывающий причинность Финслера, квадратичным конусом, в идеале аффинноинвариантным образом. Однако, мы доказываем, что на множестве выпуклых конусов не существует аффинно-ковариантной характеристики, такой как объем. Поэтому любое приближение не обязательно является аффинно-инвариантным.
\end{abstract}

Ключевые слова: геометрия пространства-времени, финслеровы пространства, причинность.

Дата поступления в редакцию: 06.12.2019 